\title{
Rehabilitación con realidad virtual en pacientes pediátricos con hemofilia. Estudio retrospectivo
}

\author{
Virtual reality rehabilitation in paediatric patients with hemophilia. Retrospective study \\ Castillo-Martínez ID, * Bremer-Aztudillo AL,* Velázquez-Marmolejo L,* \\ Moreno-González AM, ${ }^{*}$ Belmont-Sánchez J§
}

Hospital Infantil de México Federico Gómez.

\begin{abstract}
RESUMEN. Introducción: La afección articular es una complicación frecuente de la hemofilia. Los programas de realidad virtual ofrecen al paciente pediátrico un esquema dinámico con máximo beneficio funcional. El objetivo de este trabajo es evaluar el efecto de un programa de rehabilitación combinado con terapia convencional y realidad virtual sobre la función articular en pacientes con artropatía hemofílica y el impacto sobre el patrón de la marcha. Material y métodos: Se evaluaron 11 pacientes pediátricos con hemofilia A o B y antecedente de artropatía hemofílica en grado I o II. Se sometieron a un programa de rehabilitación de ocho semanas con Xbox Kinect ${ }^{\mathrm{TM}}$ y mecanoterapia. Se utilizó el laboratorio de análisis de movimiento para evaluar el patrón de marcha y la escala de salud articular al inicio y término del programa. Resultados: Se redujo 50\% $(\mathrm{p}=0.000)$, $68 \%(\mathrm{p}=0.016)$ y $48 \%(\mathrm{p}=0.000)$ el puntaje de la escala de salud total articular (STA), puntaje marcha global (PMG) y puntaje total de la escala de salud articular (P-HJHS-T) respectivamente. Se observó disminución en la fase de apoyo y zancada derecha y aumento en cadencia, longitud de paso, zancada izquierda y fase de balanceo. Conclusiones: La mejoría global de los pacientes nos hace concluir que este estudio piloto abre una ventana de oportunidades para continuar el estudio de un programa integral de rehabilitación con base en el uso de la realidad virtual en pacientes pediátricos con artropatía hemofílica.
\end{abstract}

Palabras clave: Hemofilia, rehabilitación física, realidad virtual, hemartrosis, artropatía hemofílica, marcha.
ABSTRACT. Introduction: Joint affection is a frequent complication of hemophilia. Virtual reality programs offer the patient a dynamic plan with maximum functional benefit. The objective of this work is to evaluate the effect of a rehabilitation program combined with conventional therapy and virtual reality on joint function, in patients with hemophilic arthropathy and the impact on the gait. Material and methods: Eleven pediatric patients with hemophilia and history of hemophilic arthropathy grade I or II were evaluated. An 8-week rehabilitation program was carried out with Xbox Kinect ${ }^{\mathrm{TM}}$ and mechanotherapy. Movement analysis laboratory to evaluate the gait pattern and the Joint Health Scale was performed at the beginning and end of the program. Results: A reduction of $50 \%(p=0.000)$, $68 \%(\mathrm{p}=0.016)$ and $48 \%(\mathrm{p}=0.000)$ were observed in the Total Joint Health score (STA), Global Gait Score (PMG) and Total Score of the Joint Health Scale (P-HJHS-T) respectively. There is a decrease in the support phase and right stride, and increase in cadence, step length, left stride and swing phase. Conclusions: The overall improvement of the patients makes us conclude that this pilot study opens a window of opportunity to continue the study of an integral rehabilitation program based on the use of virtual reality in pediatric patients with hemophilic arthropathy.

Keywords: Hemophilia, physical rehabilitation, virtual reality, hemarthrosis, hemophilic arthropathy, walking.

\section{Nivel de evidencia: III}

* Servicio de Hematología.

* Unidad de Investigación en Epidemiología Clínica, UNAM.

$\S$ Servicio de Rehabilitación.

Hospital Infantil de México Federico Gómez (HIMFG), Ciudad de México.

\section{Correspondencia:}

Alhelí Lucía Bremer-Aztudillo

Unidad de Epidemiología Clínica UNAM/HIMFG Calle Doctor Márquez Núm. 162, Col. Doctores, CP 06720, Alcaldía Cuauhtémoc, Ciudad de México. E-mail: alhelí_bremer@hotmail.com 


\section{Introducción}

La hemofilia es un trastorno hemorrágico congénito ligado al cromosoma $\mathrm{X}$ causado por una deficiencia del factor VIII de la coagulación (FVIII) en la hemofilia A o factor IX (FIX) en la hemofilia B. . $^{1,2}$

La afección articular es una de las complicaciones más frecuentes en la hemofilia, ${ }^{3,4,5}$ los factores que contribuyen al daño de la articulación incluyen hemartrosis recurrentes, inflamación sinovial e hipertrofia de tejidos blandos, que en última instancia conducen a pérdida del espacio articular, destrucción del cartílago y limitación funcional. ${ }^{6,7,8}$

En general, la gravedad del sangrado está correlacionada inversamente con el nivel del factor de coagulación. Los casos de hemofilia grave (nivel de factor $<1 \%$ ) sufren hemorragias espontáneas, mientras que en la hemofilia leve (nivel de factor $>5 \%$ ) el sangrado sólo se produce después de un traumatismo grave o cirugía. Las hemorragias espontáneas se producen principalmente en articulaciones como rodilla, tobillo, cadera y codo. ${ }^{9}$

En 1977, Arnold y Hilgartner diseñaron una escala de evaluación clínico-radiológica que clasifica la artropatía hemofílica en cinco etapas. ${ }^{10}$ Después, en 2006 se realizó la primera versión de la escala de salud articular en hemofilia (HJHS, por sus siglas en inglés), la cual evalúa el daño articular en niños con hemofilia; ${ }^{11}$ en el 2011, el grupo internacional para el estudio de la profilaxis (IPSG, por sus siglas en inglés) actualizó la HJHS (versión 2.1), esta escala consta de ocho elementos por articulación (más el análisis de la marcha que se analiza globalmente), evaluando: 1) inflamación de la articulación; 2) duración de la inflamación; 3) atrofia muscular; 4) fuerza; 5) crepitantes en el movimiento; 6) pérdida de flexión; 7) pérdida de extensión; y 8) dolor articular. El rango de puntaje completo de la HJHS versión 2.1 es de 0 a 124 puntos $(0-20$ puntos por cada una de las seis articulaciones evaluadas, más 4 puntos por la evaluación general de la marcha), entre mayor sea el puntaje, ma- yor es la afección articular, ${ }^{12}$ la única desventaja de la escala HJHS es que es operador-dependiente.

El laboratorio para el análisis del movimiento (laboratorio de marcha) ha demostrado ser una herramienta de gran utilidad en medicina por su importancia para el diagnóstico de alteraciones de la marcha y la evaluación de resultados de tratamientos de varias patologías, ya sean quirúrgicos o de otro tipo, así como para analizar las alteraciones en el patrón de marcha secundarias a alteraciones musculoesqueléticas o neurológicas. ${ }^{13}$ En el análisis de marcha se obtienen y analizan distintos valores espacio temporales que permiten un análisis objetivo del impacto de los ejercicios de rehabilitación en la recuperación de la movilidad articular, dentro de los cuales destacan: la duración del ciclo de la marcha, así como cada una de las fases de ésta; además de obtener el largo de paso, el largo de la zancada, ancho de paso y ángulo de paso, entre otras. ${ }^{14}$

La importancia del abordaje integral de un programa de rehabilitación en los sujetos con hemofilia se sustenta en un manejo completo que deberá abarcar dos componentes: el componente mecánico de la articulación y las alteraciones neurológicas secundarias (equilibrio, coordinación, propiocepción).

La rehabilitación con base en realidad virtual $\mathrm{Kinect}^{\mathrm{TM}}$ incluye una reeducación en los tres planos de movimiento y cinemática, mejora la planeación de movimiento, por lo cual reduce el riesgo de hemartrosis y mejora el patrón de movimiento, incluyendo la marcha, del mismo modo reduce las barreras para realizar ejercicios de rehabilitación; ${ }^{15}$ los ejercicios convencionales son repetitivos, por lo que los casos pierden interés y no completan el proceso de rehabilitación, especialmente en pacientes pediátricos; los programas de realidad virtual le ofrecen al individuo el beneficio para realizar un programa dinámico con máximo beneficio funcional, así como una mayor motivación para ejecutarlos. ${ }^{16,17,18}$ En los últimos años, la realidad virtual ha ganado terreno y se ha usado para la rehabilitación en adultos con enfermedad vascular cerebral; sin embargo, en pacientes pediátricos con hemofilia no se ha extendido su uso. ${ }^{19}$

\begin{tabular}{|c|c|c|c|}
\hline Juego & Duración (min) & $\begin{array}{l}\text { Rango de } \\
\text { movilidad }\end{array}$ & Movimiento \\
\hline Boliche & $7-10$ & Anteroposterior & $\begin{array}{l}\text { - Movimiento activo de extremidades superiores* (rotación interna de hombro) } \\
\text { - Flexión, extensión y lateralización de tronco } \\
\text { interna de cadera; flexión y extensión de rodilla; flexión y extensión de tobillo) }\end{array}$ \\
\hline Tenis & $7-10$ & $\begin{array}{l}\text { Lateral derecha- } \\
\text { izquierda }\end{array}$ & $\begin{array}{l}\text { Extremidades superiores* (rotación externa de hombro, flexión y extensión de codo, } \\
\text { supinación de antebrazo, pronación, flexión y extensión de muñeca) } \\
\text { - Rotación de tronco } \\
\text { - Cambio de los vectores de fuerza y soporte alternado en las extremidades inferiores para } \\
\text { estabilización }\end{array}$ \\
\hline Escalar & $7-10$ & Superior-inferior & $\begin{array}{l}\text { Extremidades superiores* (extensión flexión de hombro, aducción, externa y rotación } \\
\text { interna; pronación y supinación de antebrazo) } \\
\text { - Rotación, flexión, extensión y lateralización de tronco } \\
\text { - Extremidades inferiores con movimiento alternado del patrón del flexor y extensor } \\
\text { coordinado }\end{array}$ \\
\hline
\end{tabular}




\section{Tabla 2: Programa de rehabilitación en casa.}

\begin{tabular}{|c|c|c|}
\hline $\begin{array}{l}\text { Thera- } \\
\text { Band } \\
\text { Amarilla }\end{array}$ & $\begin{array}{l}1 \text { min o } 40 \\
\text { repeticiones } \\
\text { por grupo } \\
\text { muscular }\end{array}$ & $\begin{array}{l}\text { Extremidades superiores* } \\
\text { Extremidades Inferiores** }\end{array}$ \\
\hline Pesas $(0.5$ & 3 repeticiones & Extremidades Superiores* \\
\hline у $0.75 \mathrm{~kg}$ ) & $\begin{array}{l}\text { de } 1 \text { min } \\
\text { por grupo } \\
\text { muscular/2 } \\
\text { min de } \\
\text { descanso }\end{array}$ & Extremidades Inferiores** \\
\hline Bastón & $5 \mathrm{~min}$ & $\begin{array}{l}\text { Movimientos combinados de la cintura } \\
\text { escapular flexión-extensión, abducción- } \\
\text { rotación externa, abducción-rotación } \\
\text { interna, abducción-flexión }\end{array}$ \\
\hline Aro & $5 \mathrm{~min}$ & $\begin{array}{l}\text { Patrones de rotación que combinan } \\
\text { flexión-extensión de la cintura escapular, } \\
\text { rotación interna, rotación externa }\end{array}$ \\
\hline
\end{tabular}

\section{Material y métodos}

Se realizó un estudio retrospectivo comparativo. Se tomaron expedientes de la Clínica de Hemofilia del Hospital Infantil de México Federico Gómez, que participaron en la terapia con realidad virtual entre el año 2015 y 2017, con los siguientes criterios de inclusión: pacientes entre seis y 18 años de edad, cualquier tipo de hemofilia, con artropatía hemofílica grado I o II según escala de Arnold-Hilgartner, con evaluación inicial y final de la escala HJHS versión 2.1, el programa integral de rehabilitación de ocho semanas (notas de rehabilitación de cada sesión y la copia del carnet de ejercicios en casa) y el análisis del movimiento (laboratorio de marcha) antes y después del programa de las sesiones de rehabilitación.

Los sujetos con hemartrosis aguda o con inhibidores del factor VIII y IX fueron excluidos del estudio al igual que los que no cumplieron el régimen de ocho semanas del programa de rehabilitación.

El programa integral de rehabilitación de ocho semanas consistió en: dos sesiones semanales intrahospitalarias de 30 minutos con el aparato de realidad virtual, evaluando tres planos de movimiento para mejorar la coordinación motora gruesa, la propiocepción y el equilibrio dinámico usando el videojuego Microsoft Xbox One Kinect Sport Rivals $^{\mathrm{TM}}$ en nivel intermedio, bajo la supervisión del fisioterapeuta. Los pacientes recibieron profilaxis una hora antes de cada sesión de realidad virtual con intención de mantener los niveles de factor por encima de 50-60\% ( $\mathrm{Ta}$ bla 1). Lo anterior acompañado de un programa de rehabilitación en casa que consistió en una sesión domiciliaria de 30 minutos diarios de mecanoterapia definida por el Servicio de Rehabilitación (Tabla 2). Los datos se recabaron en formatos físicos de la escala de Arnold-Hilgartner (Apéndice 1) ${ }^{20}$ y después se utilizó Microsoft Office Ex-
$\mathrm{cel}^{\mathrm{TM}}$ para conformar una base de datos. El análisis estadístico se realizó con el programa SPSS v. 26.0, en donde se comprobó la normalidad de la muestra estadística y se realizó la prueba t de Student para muestras relacionadas para encontrar diferencias entre el antes y después de las mediciones del laboratorio de análisis de movimiento y de las escalas de salud articular HJHS versión 2.1, dado que $\alpha$ $=0.05 \%$, con un intervalo de confianza de $95 \%$.

\section{Resultados}

Se evaluaron 20 expedientes, de los cuales sólo 11 cumplieron con todos los criterios de inclusión, con edad media de $9.5 \pm 3$ años, la extremidad más afectada fue la izquierda, las articulaciones más afectadas fueron rodilla y tobillo.

Se compararon las medidas iniciales y finales de los 11 casos y se observó una reducción de $50 \%$ en la media de la salud total articular (STA) inicial y final, $68 \%$ del puntaje de marcha global (PMG) inicial y final y $48 \%$ del puntaje total de la escala de salud articular para hemofilia (P-HJHS-T) inicial y final, con un intervalo de confianza de $95 \%$ y una significancia estadística de $p=0.000, p=0.016$ y $p=0.000$ respectivamente (Tablas 3 y 4 ).

En los resultados arrojados por el laboratorio de análisis de movimiento, se observó disminución en los parámetros de fase de apoyo y zancada de pierna derecha, así como aumento en la cadencia, longitud de paso, zancada de pierna izquierda y fase de oscilación; no se observaron cambios en la velocidad promedio de la marcha.

Esto se traduce en una mejoría en el sistema de planeación del movimiento (sistema nervioso) con el aumento de la cadencia y la longitud de paso, que refleja mayor control en el

Tabla 3: Media de las mediciones iniciales y finales de los parámetros de la escala de salud articular.

Parámetro Medición inicial Medición final

Puntuación de salud total articular (STA)

Puntuación marcha global (PMG)

Puntuación total de la escala de

salud articular de hemofilia

(P-HJHS-T)

$\begin{array}{ll}9.00 \pm 2.8 & 4.55 \pm 3.8 \\ & \\ 0.73 \pm 0.78 & 0.27 \pm 0.46 \\ 9.73 \pm 3.2 & 5.09 \pm 3.7\end{array}$

$0.73 \pm 0.78$
$9.73 \pm 3.2$

$5.09 \pm 3.7$

\begin{tabular}{|c|c|c|c|c|}
\hline \multicolumn{5}{|c|}{$\begin{array}{l}\text { Tabla 4: Diferencias pareadas que reflejan la } \\
\text { significancia estadística de las mediciones de salud } \\
\text { articular mediante la prueba t de Student. }\end{array}$} \\
\hline Parámetro & Media $\pm \mathrm{DE}$ & IC 95\% & $\mathrm{t}$ & $\mathrm{p}$ \\
\hline STA inicial/STA final & $4.45 \pm 2.77$ & $2.5-6.31$ & 5.33 & 0.00 \\
\hline PMG inicial/PMG final & $0.45 \pm 0.52$ & $0.10-0.80$ & 2.88 & 0.01 \\
\hline $\begin{array}{l}\text { P-HJHS-T inicial/ } \\
\text { P-HJHS-T final }\end{array}$ & $4.63 \pm 2.87$ & $2.70-6.56$ & 5.35 & 0.00 \\
\hline
\end{tabular}


Tabla 5: Reporte estadístico de las mediciones realizadas en el laboratorio de análisis de movimiento.

\section{Parámetro}

Fase de apoyo pierna derecha (s) Fase de apoyo pierna izquierda (s) Fase de oscilación pierna derecha (s) Fase de oscilación pierna izquierda (s) Zancada pierna derecha (s)

Zancada pierna izquierda (s)

Cadencia (pasos/min)

Longitud de paso pierna derecha $(\mathrm{m})$

Longitud de paso pierna izquierda (m)

Velocidad promedio $(\mathrm{m} / \mathrm{s})$
Inicial

Final

$58.47 \pm 7.53$

$57.33 \pm 4.37$

$41.49 \pm 8.07$

$43.36 \pm 4.58$

$1.09 \pm 0.13$

$1.06 \pm 0.07$

$112.79 \pm 8.94$

$0.39 \pm 0.10$

$0.39 \pm 0.06$

$0.81 \pm 0.14$
$58.05 \pm 5.60$

$56.84 \pm 7.68$

$41.73 \pm 6.40$

$43.82 \pm 6.70$

$1.05 \pm 0.09$

$1.07 \pm 0.09$

$115.14 \pm 10.65$

$0.44 \pm 0.06$

$0.45 \pm 0.06$

$0.83 \pm 0.14$ $\mathrm{s}=$ segundos $; \min =$ minuto $; \mathrm{m}=$ metro.

sistema efector (sistema musculoesquelético), por lo cual el paciente tiene menor riesgo de presentar una nueva hemartrosis o, en caso de presentarla, puede tener una recuperación funcional más rápida. El parámetro que reflejó mejoría en el sistema efector es la longitud de paso izquierda $(p=0.005)$, esto concuerda con los resultados presentados con anterioridad respecto a la mejoría de la marcha global según la escala de salud articular para la hemofilia (HJHS) (Tabla 5).

\section{Discusión y conclusiones}

Se encontró una diferencia significativa en los valores de las medias de las escalas de salud articular de los sujetos antes y después del tratamiento. Utilizando el laboratorio de análisis de movimiento, se logró identificar mejoría en los parámetros previamente descritos, aunque solamente se logró encontrar significancia estadística en la longitud del paso $(\mathrm{p}=0.005)$. El apego a los programas de rehabilitación que se direccionan con componentes lúdicos hace que la tasa de apego al programa sea mucho mayor que con los ejercicios de rehabilitación convencionales, incluso con el apoyo y la supervisión de los padres de familia. El laboratorio de análisis de movimiento nos apoya objetivamente en la evaluación de los parámetros específicos de la marcha y, por tanto, se considera como un factor fundamental en la evaluación de futuros protocolos.

La mejoría clínica de los casos reflejada en la escala de salud articular y en el análisis de movimiento del patrón de marcha, aunado al apego de los tratamientos de rehabilitación con realidad virtual, nos hace concluir que la realidad virtual en pacientes con hemofilia es una estrategia divertida y moderna para que estos pacientes lleven a buen puerto la terapia de rehabilitación, obteniendo grandes resultados como mejoría en la marcha y disminución del número de sangrados.

\section{Agradecimientos y financiamiento}

A Novo Nordisk Hemophilia Foundation por proveer los recursos de impresión de guías de ejercicios, kits de rehabilitación y Xbox Kinect ${ }^{\mathrm{TM}}$ para la clínica de hemofilia HIMFG.

\section{Referencias}

1. Stonebraker JS, Bolton-Maggs PH, Soucie JM, Walker I, Brooker M. A study of variations in the reported haemophilia A prevalence around the world. Haemophilia. 2010; 16(1): 20-32.

2. Stonebraker JS, Bolton-Maggs PH, Michael Soucie J, Walker I, Brooker M. A study of variations in the reported haemophilia B prevalence around the world. Haemophilia. 2012; 18(3): e91-4.

3. Luck JV Jr, Silva M, Rodriguez-Merchan EC, Ghalambor N, Zahiri CA, Finn RS. Hemophilic arthropathy. J Am Acad Orthop Surg. 2004; 12(4): 234-45.

4. Muça-Perja M, Riva S, Grochowska B, Mangiafico L, Mago D, Gringeri A. Ultrasonography of haemophilic arthropathy. Haemophilia. 2012; 18(3): 364-8.

5. Ceponis A, Wong-Sefidan I, Glass CS, von Drygalski A. Rapid musculoskeletal ultrasound for painful episodes in adult haemophilia patients. Haemophilia. 2013; 19(5): 790-8.

6. Acharya SS, Schloss R, Dyke JP, Mintz DN, Christos P, DiMichele $\mathrm{DM}$, et al. Power Doppler sonography in the diagnosis of hemophilic synovitis--a promising tool. J Thromb Haemost. 2008; 6(12): 2055-61.

7. Bhat V, Olmer M, Joshi S, Durden DL, Cramer TJ, Barnes RF, et al. Vascular remodeling underlies rebleeding in hemophilic arthropathy. Am J Hematol. 2015; 90(11): 1027-35.

8. Wyseure T, Mosnier LO, von Drygalski A. Advances and challenges in hemophilic arthropathy. Semin Hematol. 2016; 53(1): 10-9.

9. Aronstam A, Rainsford SG, Painter MJ. Patterns of bleeding in adolescents with severe haemophilia A. Br Med J. 1979; 1(6161): 469-70.

10. Arnold WD, Hilgartner MW. Hemophilic arthropathy. Current concepts of pathogenesis and management. J Bone Joint Surg Am. 1977; 59(3): 287-305.

11. Hilliard P, Funk S, Zourikian N, Bergstrom BM, Bradley CS, McLimont M, et al. Hemophilia joint health score reliability study. Haemophilia. 2006; 12(5): 518-25.

12. Bladen M, Main E, Hubert N, Koutoumanou E, Liesner R, Khair K. Factors affecting the Haemophilia Joint Health Score in children with severe haemophilia. Haemophilia. 2013; 19(4): 626-31.

13. Nelson AJ. Functional ambulation profile. Phys Ther. 1974; 54(10): 1059-65.

14. Nelson AJ, Zwick D, Brody S, Doran C, Pulver L, Rooz G, et al. The validity of the GaitRite and the Functional Ambulation Performance scoring system in the analysis of Parkinson gait. NeuroRehabilitation. 2002; 17(3): 255-62.

15. Park DS, Lee DG, Lee K, Lee G. Effects of virtual reality training using Xbox Kinect on motor function in stroke survivors: a preliminary study. J Stroke Cerebrovasc Dis. 2017; 26(10): 2313-9.

16. Betker AL, Szturm T, Moussavi ZK, Nett C. Video game-based exercises for balance rehabilitation: a single-subject design. Arch Phys Med Rehabil. 2006; 87(8): 1141-9.

17. Ficklscherer A, Stapf J, Meissner KM, Niethammer T, Lahner M, Wagenhäuser $\mathrm{M}$, et al. Testing the feasibility and safety of the Nintendo Wii gaming console in orthopedic rehabilitation: a pilot randomized controlled study. Arch Med Sci. 2016; 12(6): 1273-8.

18. Messier SP, Royer TD, Craven TE, O'Toole ML, Burns R, Ettinger WH Jr. Long-term exercise and its effect on balance in older, osteoarthritic adults: results from the Fitness, Arthritis, and Seniors Trial (FAST). J Am Geriatr Soc. 2000; 48(2): 131-8.

19. Pérez-Alenda S, Carrasco JJ, Aguilar-Rodríguez M, Martínez-Gómez L, Querol-Giner M, Cuesta-Barriuso R, et al. Balance evaluation in haemophilic preadolescent patients using Nintendo Wii Balance Board $^{\circledR}$. Haemophilia. 2017; 23(1): e18-24.

20. Feldman BM, Funk SM, Bergstrom BM, Zourikian N, Hilliard P, van der Net J, et al. Validation of a new pediatric joint scoring system from the International Hemophilia Prophylaxis Study Group: validity of the hemophilia joint health score. Arthritis Care Res (Hoboken). 2011; 63(2): 223-30.

Conflicto de intereses: Los autores de este artículo declaran no tener ningún conflicto de intereses. 
Apéndice 1: Escala de salud articular en hemofilia.

Puntuación de salud articular en hemofilia 2.1 - Hoja resumen de puntuación

\begin{tabular}{|l|l|l|l|l|l|l|}
\hline & Codo izquierdo & Codo derecho & Rodilla izquierda & Rodilla derecha & Tobillo izquierdo & Tobillo derecho \\
\hline $\begin{array}{l}\text { Inflamación } \\
\text { Duración (inflamación) }\end{array}$ & $\square \mathrm{NE}$ & $\square \mathrm{NE}$ & $\square \mathrm{NE}$ & $\square \mathrm{NE}$ & $\square \mathrm{NE}$ & $\square \mathrm{NE}$ \\
\hline $\begin{array}{l}\text { Atrofia muscular } \\
\text { Complicaciones en el } \\
\text { movimiento }\end{array}$ & $\square \mathrm{NE}$ & $\square \mathrm{NE}$ & $\square \mathrm{NE}$ & $\square \mathrm{NE}$ & $\square \mathrm{NE}$ & $\square \mathrm{NE}$ \\
\hline $\begin{array}{l}\text { Pérdida de flexión } \\
\text { Pérdida de extensión }\end{array}$ & $\square \mathrm{NE}$ & $\square \mathrm{NE}$ & $\square \mathrm{NE}$ & $\square \mathrm{NE}$ & $\square \mathrm{NE}$ & $\square \mathrm{NE}$ \\
\hline $\begin{array}{l}\text { Dolor articular } \\
\text { Fuerza }\end{array}$ & $\square \mathrm{NE}$ & $\square \mathrm{NE}$ & $\square \mathrm{NE}$ & $\square \mathrm{NE}$ & $\square \mathrm{NE}$ \\
\hline \begin{tabular}{l} 
Total de articulaciones \\
\hline
\end{tabular} & $\square \mathrm{NE}$ & $\square \mathrm{NE}$ & $\square \mathrm{NE}$ & $\square \mathrm{NE}$ & $\square \mathrm{NE}$ & $\square \mathrm{NE}$ \\
\hline
\end{tabular}

$\mathrm{NE}=$ No evaluable

Suma del total de articulaciones

Puntuación de la marcha global

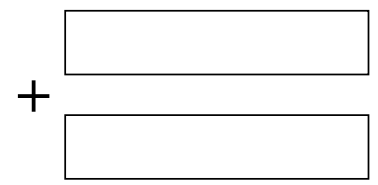

( $\square$ NE incluido en los ítems de la marcha)

Puntuación HJHS total

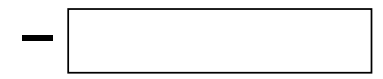

\section{Inflamación \\ $0=$ Sin inflamación \\ $1=$ Leve \\ $2=$ Moderada \\ $3=$ Intensa}

\section{Crepitantes en el movimiento}

$0=$ Ninguna

$1=$ Leve

$2=$ Intensa

\section{Pérdida de flexión}

\section{Duración}

$0=$ Sin inflamación $0<6$ meses

$1=\geq 6$ meses

Atrofia muscular
$0=$ Ninguna
$1=$ Leve
$2=$ Severa

\section{Lado contralateral:}

$0=<5$ grados

$1=$ Pérdida de 5 a $10^{\circ}$

$2=$ Pérdida de 11 a $20^{\circ}$

$3=$ Pérdida de $>20^{\circ}$
Lado contralateral:

$0=<5$ grados

$1=$ Pérdida de 5 a $10^{\circ}$

$2=$ Pérdida de 11 a $20^{\circ}$

$3=$ Pérdida de $>20^{\circ}$

\section{Pérdida de extensión (desde hiperextensión)}

Tablas normativas:

$0=$ Dentro del rango

$1=$ Pérdida de 1 a $4^{\circ}$

$2=$ Pérdida de 5 a $10^{\circ}$

$3=$ Pérdida de $>10^{\circ}$

Tablas normativas:

$0=$ Dentro del rango

$1=$ Pérdida de 1 a $4^{\circ}$

$2=$ Pérdida de 5 a $10^{\circ}$

$3=$ Pérdida de $>10^{\circ}$
Fuerza (usando la escala de Daniels \& Worthingham) Dentro del rango de movimiento disponible $0=$ Mantiene la posición de la prueba contra la gravedad con una resistencia máxima (gr. 5).

$1=$ Mantiene la posición de la prueba contra la gravedad con una resistencia moderada (pero cede cuando hay una resistencia máxima (gr. 4).

$2=$ Mantiene la posición de la prueba contra la gravedad con una resistencia mínima (gr. 3+), o mantiene la posición de la prueba contra la gravedad (gr. 3).

3 = Capaz de completar parcialmente el rango de movimiento contra la gravedad (gr. 3-/2+), 0 capaz de moverla a través del rango de movimiento con gravedad eliminada (gr. 2), o a través de gravedad parcial el rango de movimiento eliminada (gr. 2-).

4 = Indicios (gr. 1) o sin contracción muscular (gr. 0).

$\mathrm{NE}=$ No evaluable

Marcha global (caminar, escaleras, correr, salto sobre una pierna)

$0=$ Todas las habilidades se encuentran en los límites normales.

$1=$ Una habilidad no está dentro de los límites normales.

$2=$ Dos habilidades no están dentro de los límites normales.

$3=$ Tres habilidades no están dentro de los límites normales.

4 = Ninguna habilidad está dentro de los límites normales. $\mathrm{NE}=$ No evaluable

Dolor articular

$0=$ Sin dolor en el rango de movimiento activo

$1=$ Sin dolor en el rango de movimiento activo; solamente dolor

en la presión suave o en la palpación

$2=$ Dolor en el rango de movimiento activo 